\title{
LA ACEPTACIÓN DE LA DIVERSIDAD EN CUESTIÓN: EXPERIENCIAS ENRIQUECEDORAS Y ADVERSAS EN LA FORMACIÓN Y LA PRÁCTICA PROFESIONAL TEMPRANA EN PSICOLOGÍA
}

\begin{abstract}
María Inés Winkler Müller ${ }^{1}$, María Isabel Reyes Espejo ${ }^{2}$
Resumen: Ante un marco regulador ambiguo del ejercicio profesional en el país y una praxis de la psicología en el contexto de una identidad profesional difusa y límites poco establecidos con otras profesiones, se releva la importancia de conocer temas transversales que refieren a la relación con el otro. En este estudio se indagó en la percepción de un grupo de estudiantes y psicólogos jóvenes acerca de la formación profesional recibida y sus primeras experiencias laborales, específicamente en temas de interculturalidad y ética; así como también sobre fortalezas y debilidades de la formación recibida. Se realizó 18 entrevistas individuales y dos grupos focales con 12 participantes de dos universidades estatales $(\mathrm{N}=30)$. Se aplicó un análisis categorial intersubjetivamente, del cual emergieron cuatro categorías centrales: contexto, experiencias formadoras y adversas en la formación, noción de interculturalidad y sugerencias para la formación en ética e interculturalidad. Destaca en los resultados el relato de experiencias formadoras y de experiencias adversas en la formación recibida, así como una noción de interculturalidad centrada en situaciones concretas. Se concluye respecto de algunos desafíos que enfrenta la formación de psicólogos y psicólogas en Chile.
\end{abstract}

Palabras clave: ética en psicología, interculturalidad, formación en psicología, profesionales

Diversity acceptance in question: enriching and adverse experiences in training and early professional practice in psychology

\begin{abstract}
Faced with an ambiguous regulatory framework of professional practice in the country; and a practice of psychology in the context of a fuzzy professional identity and without clear boundaries with other professions, the importance of knowing crosscutting issues that concern the relationship with the other is relieved. This study explored the perception a group of students and novel psychologists about the training received and their first work experience, specifically in issues of interculturality and ethics; as well as on strengths and weaknesses of the training received. Eighteen individual interviews and two focus groups with 12 participants, from two state universities was conducted $(\mathrm{N}=30)$. A intersubjectively categorical analysis was applied and four main categories emerged: context, positive, and adverse experiences in training, notion of interculturality, and suggestions for training in ethics and interculturality. We conclude on some challenges facing the formation of psychologists in Chile.
\end{abstract}

Key words: ethics in psychology, interculturality, training in psychology, professionals

\section{A aceitaçáo da diversidade em questão: experiências enriquecedoras e adversas na formaçáo e na prática profissional} precoce em psicologia

Resumo: Perante um marco regulador ambíguo do exercício profissional no país; e uma práxis da psicologia no contexto de uma identidade profissional difusa e limites pouco estabelecidos com outras profissóes, é revelada a importância de conhecer temas transversais que se referem à relação com o outro. Neste estudo, indagou-se na percepçáo de um grupo de estudantes e psicológos/as jovens acerca da formação profissional recebida e suas primeiras experiências de trabalho, especificamente sobre questóes de interculturalidade e ética; assim como os pontos fortes e fracos do formação recebida. Foram realizadas 18 entrevistas individuais e dois grupos focais com 12 participantes de duas universidades públicas $(\mathrm{N}=30)$. Aplicou-se uma análise categórica intersubjetivamente, da qual emergiu quatro categorias principais: contexto, experiências formadoras e adversas na formação, noção de interculturalidade e sugestôes para a formaçáo em ética e interculturalidade. Destaca-se nos resultados o relato de experiências formadoras e experiências adversas na formação recebida; bem como uma noção de interculturalidade, centrada em situações concretas. Conclui-se a respeito a alguns desafios que enfrenta a formação de psicólogos e psicólogas no Chile.

Palavras-chave: ética em psicologia, interculturalidade, formação em psicologia, profissionais

\footnotetext{
${ }^{1}$ Escuela de Psicología, Universidad de Santiago de Chile, Chile

Correspondencia: maria.winkler@usach.cl

${ }^{2}$ Escuela de Psicología, Pontificia Universidad Católica de Valparaíso, Chile
} 


\section{Introducción: ética e interculturalidad}

Enseñar siempre: en el patio y en la calle como en la sala de clases. Enseñar con la actitud, el gesto y la palabra

(Gabriela Mistral, Desolación, 1923).

En el estudio de las profesiones, el campo de la ética ha cobrado interés en la medida que los cambios tecnológicos, medioambientales y políticos en el mundo han puesto en evidencia que las prácticas emanadas del conocimiento científico obstaculizan tiempos de reflexión adecuados para evaluar los fines, valores y principios últimos a los cuales sirven. Más aún si consideramos que la ética de una profesión no puede ser considerada como "dada", sino que se encuentra en un constante proceso de construcción, influido por el devenir histórico, se vuelve todavía más importante incorporar exploraciones empíricas que ayuden a levantar e interpretar los procesos de interacción que se dan en los grupos profesionales, incluyendo procesos biográficos identitarios(1).

En el caso particular de la psicología, el análisis de la ética en la praxis profesional resulta especialmente importante, puesto que la identidad profesional y los límites de la psicología con otras profesiones aún no están bien establecidos, ni contamos en Chile con una ley della psicólogola que describa sus ámbitos y límites del quehacer profesional $(2-4)$.

Promisoriamente, algunos eventos concretos han apuntado al aumento en la toma de conciencia de la relevancia de la ética en la formación y en la práctica profesional en el país. En 1996 se creó una nueva Comisión de Etica en el Colegio de Psicólogos de Chile (A.G.), junto a la elaboración de un nuevo Código de Ética, tres años más tarde (1999). Subsecuentemente, es posible identificar un conjunto de acciones para la difusión y educación en la normativa ética vigente, así como la promoción del aumento de conciencia en temas éticos (incorporación del Código y de artículos o documentos pertinentes o sobre temas específicos en la web del Colegio, edición de boletines especiales con los fallos de la Comisión, entre otros). Asimismo, la principal fuente estatal de financiamiento para la investigación científica, el Consejo
Nacional de Investigación, Ciencia y Tecnología (CONICYT), ha incorporado en sus bases exigencias éticas, acordes con la ley 20.120 (Sobre la Investigación Científica en Seres Humanos, Genoma y Prohíbe la Clonación Humana), todas seńales de un renovado interés y reconocimiento de la centralidad de los temas éticos en la formación profesional y científica.

En el ámbito de la formación profesional, se reporta una incorporación creciente de asignaturas que abordan temas de ética en las mallas curriculares de la mayoría de las escuelas de psicología, acompańada de la explicitación en perfiles profesionales de su importancia o mención como objetivo transversal(5). Más recientemente, algunas líneas de investigación dan cuenta de un escenario complejo, en el que se evidencia una percepción "negativa" de los profesionales chilenos frente al Código de Ética Profesional $(6,7)$ y, además, se constata la necesidad de ampliar el alcance de este último para hacerlo operativo en otras áreas del ejercicio profesional, como el campo comunitario, jurídico, laboral-organizacional y educacional $(5,8-10)$.

La constatación de ausencia de normas específicas que faciliten el enfrentamiento de la ética profesional en campos emergentes en el ejercicio de la psicología en nuestro país y en disputa con otras profesiones, añade una mayor complejidad al momento de pensar un ejercicio ético de la profesión. Particularmente, se ha puesto énfasis en promover el desarrollo de una formación ético-profesional subrayando la inserción de estrategias educativas tempranas, orientadas a la incorporación de la ética de manera integral, es decir, como personas y como ciudadanos/as, pero también contemplando y entendiendo el proceso formativo como un componente de la identidad profesional(11). Ello es consistente con una comprensión posmoderna de la ética, traducida en una demanda que busca reestablecer su significado en el plano de "la proximidad", basada en el respeto y compromiso de la intersubjetividad devenida en responsabilidad individual. Es decir, una ética ciudadana que permita integrar progresivamente una reflexión acerca de las tareas pendientes para la sociedad en su conjunto(7). 
Desde principios del 2000, las orientaciones internacionales de la formación ético-moral en el plano universitario se han dirigido a los tema de ciudadanía y responsabilidad social, traducidos en planteamientos que abogan, entre otros ejemplos, por una formación en valores(12), una ética cívica o de los mínimos(13) o por una ética intercultural(14). En dicho contexto, las aproximaciones apuestan por la transversalidad de ciertos contenidos que debieran incorporar los futuros profesionales en cuanto personas, ciudadanas y actores sociales relevantes(11).

Además, de manera complementaria, la formación en el campo de la ética profesional se dirige a responder las inquietudes relativas al buen desempeño profesional, es decir, a reflexionar sobre las consecuencias de la actuación profesional antes de llevarla a cabo(15). Para ello, de acuerdo al planteamiento de Pasmanik \& Winkler(11), resulta relevante incorporar los códigos de ética profesional en un marco reflexivo y aplicado (con un fuerte énfasis en las demandas de su uso práctico y como principios orientadores en su accionar profesional) $\mathrm{y}$, también, considerar los procesos que acompañan la adquisición de una identidad profesional en la práctica, es decir, cuando una persona es capaz de integrar los conocimientos y habilidades de la profesión, adoptando los valores y actitudes que la caracterizan(16). Esto último se relaciona contemporáneamente con las demandas de la actuación profesional en un contexto globalizado y que requiere de la aplicación de conocimientos, normas, valores en forma coherente con la cultura de la profesión frente a situaciones éticamente complejas.

Es así como en el plano de la reflexión ético profesional de la psicología es posible identificar algunos trabajos nacionales e internacionales ${ }^{3}$ que cuestionan más directamente la posición éticopolítica de este grupo profesional frente a la vulneración de los derechos humanos(17-19). Así, la discusión en el plano de la ética se ha traslado a problemas concretos, mostrando cómo la actuación profesional requiere de una preparación y entrenamiento que permita abordar integralmente temas de diversidad, género, interculturalidad, derechos medioambientales y derechos humanos.

\footnotetext{
${ }^{3}$ Un ejemplo paradigmático es la participación de psicólogos en interrogatorios en Guantánamo(17).
}

La dimensión intercultural en el marco de la ética responde a la comprensión e integración de nuevos desafíos asociados a los procesos de globalización. En el plano de la bioética, se ha puesto de relieve una serie de consideraciones relativas al respeto a las tradiciones comunitarias, en los planos social, cultural y ético(20). Así, los valores de la globalidad, como de la diversidad, han cobrado igual relevancia bajo el reconocimiento de la necesidad de generar "un entendimiento social para salvaguardar la diversidad cultural" (14:8). La estrategia formativa, en este contexto, requiere del entrenamiento de profesionales capaces de internalizar que las características culturales particulares son permeables y que todo ser humano tiene derecho al ejercicio de su libertad(21). Concretamente, desde la perspectiva de una ética intercultural, se promueve un desarrollo crítico ante posturas etnocentristas y se promueve el desarrollo de nuevos roles ciudadanos frente a los retos de una sociedad globalizada(9-24).

Entre las aproximaciones y modelos para la enseńanza de la ética en psicología existen aproximaciones que abogan por una formación integrada a una lógica de aculturación, es decir, comprendiéndola en el marco de una cultura particular referida a su quehacer como práctica profesional y disciplina científica(24). Desde esta perspectiva, se plantea que cualquier incorporación exitosa de la ética profesional dependerá de la integración de los valores personales y, por tanto, demanda un trabajo de conocimiento de la ética personal de origen. Los procesos de enseńanza-aprendizaje de la ética en psicología requieren de un abordaje que enfatice la responsabilidad del profesional con su sociedad de origen, pero que al mismo tiempo sea capaz de incorporar una perspectiva intercultural para la comprensión de fenómenos que pueden estar más alejados de su experiencia cercana(11).

Considerando que actualmente en Chile existen escasas aproximaciones empíricas que nos informen acerca del estado de la ética en términos de características identitarias de la profesión y, en particular, de las problemáticas que los profesionales y estudiantes de psicología identifican como relevantes en el campo de la ética $(11,9,22)$ decidimos indagar en sus percepciones, incorporando como una dimensión central aquello que ha impactado en sus trayectorias formativas. Asumimos, en este 
Tabla No 1: Sexo y especialidad de estudiantes y psicólogos entrevistados $\left({ }^{*}\right)$.

\begin{tabular}{|l|l|l|l|l|l|l|l|l|l|}
\hline & \multicolumn{3}{l|}{ Entrevistas } & \multicolumn{3}{l|}{ Grupos focales } & Total \\
\hline & EF & EM & PF & PM & EF & EM & PF & PM & \\
\hline Psicología Clínica & & & 2 & 2 & 4 & & & & $8(26,6 \%)$ \\
\hline Psicología Educacional & 2 & & 2 & 1 & 1 & 1 & & & $7(23,4 \%)$ \\
\hline Psicología Organizacional & & & 4 & 2 & 1 & 2 & & & $9(30 \%)$ \\
\hline Psciología Comunitaria & & & 1 & 2 & & 3 & & & $6(20 \%)$ \\
\hline Total & 2 & & 9 & 7 & 6 & 6 & & & 30 \\
\hline
\end{tabular}

*EF: estudiante femenino; E: estudiante masculino; PF: profesional femenino; PM: profesional masculino.

sentido, que la formación en ética profesional es un proceso que debe iniciarse desde los primeros años de la carrera universitaria contemplando la identificación de aspectos contextuales, es decir, que contribuyan a situar y relevar aquellas situaciones que son "sentidas" desde un ámbito próximo al ejercicio profesional.

En el contexto de una investigación mayor (SOC 1110) nos aproximamos a las experiencias de psicólogos/as y estudiantes de psicología chilenos/as en las dimensiones de ética e interculturalidad con el propósito de contribuir al fortalecimiento de la formación ética e intercultural de profesionales empoderados con conocimientos y habilidades para la toma de decisiones éticas y para el agenciamiento en temáticas de orden ético e intercultural. Específicamente, en este trabajo nos planteamos como objetivo identificar y caracterizar las experiencias que los propios entrevistados narran al indagar sobre la formación profesional en ética.

\section{Método}

Desde un marco constructivista-interpretativo, se analizaron 18 entrevistas realizadas a psicólogos y psicólogas y estudiantes en práctica de dos universidades del Consorcio de Universidades Estatales, y dos grupos focales con cinco y siete practicantes respectivamente, de ambas universidades. Es decir, participaron 30 personas, entre julio de 2013 y julio de 2014. La selección de participantes fue a partir de contactos personales del equipo de investigación y siguiendo la estrategia de la bola de nieve o muestreo en cadena(25), teniendo presente las distintas alternativas de formación que las universidades ofrecen (ver tabla No 1). Se aplicó un procedimiento de consentimiento informado, que quedó registrado en un acta, sancionada previamente por el Comité de Ética de la Universidad de Santiago de Chile. Se comprometió absoluta confidencialidad en el manejo de los datos y devolución de información a las instituciones participantes.

Las entrevistas fueron realizadas a partir de una pauta flexible de preguntas, con la cual se indagó en la formación recibida y las primeras experiencias profesionales en temas de interculturalidad y ética, así como también sobre fortalezas y debilidades de la formación recibida. Luego, siguiendo las propuestas metodológicas del método de la teoría fundamentada empíricamente(24), se procedió al análisis inductivo de las transcripciones, con un análisis intersubjetivo que se tradujo en la elaboración de categorías centrales construidas en forma mixta, desde las preguntas orientadoras y desde las respuestas analizadas inductivamente.

\section{Resultados: experiencias éticas y no éticas en la formación y práctica profesional temprana}

En la presentación de resultados se ha incorporado citas textuales de las entrevistas, identificando el número de entrevista, sexo y la especialidad de la psicología en que se está formando, o se desempeña la persona entrevistada ${ }^{4}$.

\footnotetext{
${ }^{4}$ Aunque la formación de pregrado en psicología en Chile está definida como generalista, muchas universidades ofrecen una profundización en una o dos áreas de la psicología, ya sea a partir del cuarto año de la carrera o particularmente, en la práctica profesional. A ello se refieren las personas cuando hablan de la especialidad en que se formaron.
} 
Del análisis intersubjetivo de las entrevistas y los grupos realizados, emergieron en primer lugar antecedentes del contexto, tanto global del país como del ámbito de la formación universitaria recibida, los que enmarcan las experiencias reseñadas como relevantes. De forma saliente destacó la influencia de experiencias concretas, identificables, específicas, que referían a las preguntas centrales de la investigación. Tales experiencias fueron categorizadas, para fines de este trabajo, como experiencias enriquecedoras (éticas) o adversas (no éticas), que marcaron no solo la narrativa de las personas entrevistadas, sino como experiencias subjetivamente significativas, centrales, en su concepción de lo correcto e incorrecto, tanto en la formación como en sus experiencias profesionales. Estas experiencias pueden ser desagregadas en dos grandes grupos: experiencias positivas, altamente valoradas, formadoras o experiencias negativas, de franca discriminación o de omisión de temas considerados relevantes, tanto en la formación como en la práctica profesional. Una tercera categoría central remite específicamente a la noción de interculturalidad; y la última contiene sugerencias para la formación.

Para resumir los resultados presentamos a continuación una tabla que resume dichas categorias junto a sus propiedas y ejemplos de extractos de entrevistas (tabla No 2):

En síntesis, el contexto descrito sería abiertamente discriminador. Respecto de la formación propiamente tal, nuestros entrevistados identifican experiencias que los marcaron en su trayectoria universitaria, tanto enriquecedoras como adversas. La noción de interculturalidad obtenida en las entrevistas es restringida, dando cuenta de la misma queja de los entrevistados de las omisiones que al respecto apreciaron en su formación.

\section{Conlusiones}

La reconstrucción de las experiencias —éticas y no éticas-, así como el señalamiento de las ausencias y omisiones en la formación recibida, dan cuenta de una mirada crítica de parte de los participantes en este estudio. Tal visión refiere tanto a ciertas condiciones del contexto nacional y del contexto de formación, como a factores que afectan las apreciaciones respecto de la formación recibida y la experiencia profesional temprana. Destacan las menciones a un contexto discriminador, de una gran desigualdad social, y prejuicioso ante la diversidad cultural producto de las inmigraciones.

En cuanto a la formación recibida, se destacan experiencias subjetivamente significativas, centrales, en su concepción de lo correcto e incorrecto; experiencias reconocidas como formadoras, que dejaron marcas positivas en quienes las vivieron y que son recordadas y valoradas cuando se está en el mundo profesional.

Emerge como desafío pendiente la inclusión transversal de ética e interculturalidad en la formación. En particular, pareciera que la exposición a situaciones no éticas y una formación insuficiente son significadas como experiencias que no los/as preparan para el enfrentamiento de transgresiones y dilemas éticos en la práctica profesional temprana. Asimismo, destaca la omisión de aspectos sociales estructurales en el análisis y comprensión de las problemáticas enfrentadas en el contexto social y laboral.

La diversidad de experiencias adversas y negativas relatadas por los entrevistados también podría estar dada por los contextos de enfrentamiento a una realidad ante la cual se sienten inseguros y poco preparados. Desde esta perspectiva se puede comprender también sus fuertes críticas a la formación en ética recibida y a la propia labor profesional de los psicólogos en el país.

La noción de interculturalidad obtenida en las entrevistas puede ser considerada como restringida, dando cuenta de la misma queja referida a las omisiones que apreciaron en su formación. Aunque algunas de las personas entrevistadas poseen una noción de interculturalidad amplia, la mayoría asocia el concepto o bien con pueblos originarios o bien con necesidades educativas especiales.

Muchos de ellos refieren que su formación en el campo de la ética e interculturalidad han sido insuficientes; no obstante, sí dan muestra de haber desarrollado una cierta sensibilidad ética para identificar, describir y caracterizar una serie de problemas de tipo ético e intercultural en sus contextos formativos y laborales. 
Además, la relación con el contexto socio-histórico nos permite hipotetizar respecto de las situaciones contingentes que los profesionales noveles han debido y deben enfrentar, evidenciando la necesidad de actualizar nuestros abordajes en términos de los casos y situaciones a los que se los pudieran enfrentar desde una perspectiva formativa en el campo de la acción reflexiva. Esto puede relacionarse con la necesidad de actualizar el Código de Ética del Colegio de Psicólogos de Chile, vigente desde 1990 y criticado por estar principalmente orientado a la práctica clínica. Pensamos que parte de los resultados son atribuibles al contexto posmoderno, en que se privilegia la mirada subjetiva y la generación de códigos particulares, que se rearticulan según las circunstancias e influencias políticas e ideológicas $(7,9,22)$.

Los resultados presentados cuestionan, al menos parcialmente, el discurso dominante en la formación profesional de psicólogos. Por ejemplo, un conjunto de universidades chilenas ha consen- suado que quien egresa de la carrera(23) debe ser capaz de desplegar una serie de competencias específicas, con creatividad y flexibilidad, en los diferentes ámbitos de desempeño, en línea con normativas éticas, legales y aspectos culturales propios de cada contexto(2). Tal declaración no se corresponde con la visión crítica de nuestros entrevistados, quienes denuncian desafíos pendientes en su formación, que den cuenta de lo que Bilbeny(14) denomina "valores interculturales básicos" desde el punto de vista ético: aceptación y respeto mutuo.

\section{Agradecimientos}

Resultados parciales del Proyecto Anillos SOC 1110 "Iluminando un Dilema Educacional en la Complejidad de un Mundo Multicultural: Fortalecimiento de la Formación en Ética e Interculturalidad en Estudiantes Universitarios y Profesionales", financiado por CONICYT. 
Tabla No 2: Resultados del análisis descriptivo, incluyendo categorías centrales, subcategorías, propiedades y extractos de las entrevistas.

1. CONTEXTO: Antecedentes que afectaron la formación o influyen de manera relevante en la práctica profesional, en la dimensión ética.

1.1. Contexto nacional: Características identificadas como propias del país.

1.1.1. Crítica a la participación politica: Uno tiene que sufrir las repercusiones de una sociedad muy despolitizada, discriminatoria de las posiciones (...) no puedo dejar de nombrar la dictadura, que lamentablemente lo que hizo la dictadura en Chile fue quebrar la relación simbólica y la valoración social de la militancia social y de la militancia política; la militancia política uno la tiene más o menos fija, los partidos, la militancia en colectivos; pero la militancia social también, que tenía que ver con cómo uno participaba de juntas de vecinos, de la federación de estudiantes (E 1, Comunitario).

1.1.2. Discriminación: La discriminación, ya sea por lugar de residencia, que también se suele hacer; por clase social, porque se hace harto y por género y por edad, o sea, todo tipo de discriminación, se hace bastante (E 9 , Organizacional).

1.1.3. Prejuicios ante la diversidad: Vas caminando y te encuentras conversaciones en inglés, más allá pasa un haitiano, te encuentras con los colombianos conversando, más allá hay un español o un argentino, es maravilloso pero no estamos preparados, tenemos que enfrentar un montón de prejuicios internos (E 9, Organizacional).

1.2. Contexto formativo: Respecto de la formación recibida, encontramos principalmente referencias a temas ausentes en ella.

1.2.1. Ausencia de crítica a la desigualdad social: Un tema que habría que ahondar en temas de la ética es ser abiertamente crítico sobre la situación estructural chilena de desigualdad, donde no nos podemos hacer los lesos y tenemos que ver cómo nos situamos sobre eso. Yo creo que eso también dentro de mi formación no estaba, sí en algunos ramos de social (...) siendo que es un tema que tiene que ser transversal (E3, Comunitaria).

1.2.2. Ausencia de formación en diversidad: En la escuela no, de hecho se toca muy poco esos temas y de hecho la diversidad no es parte de esta escuela. Es súper rígida en cuanto a su malla y no hay espacios para eso, de hecho lo que he aprendido es porque uno va recorriendo otros espacios y quiere salir de la universidad un poco (...). Por ejemplo, yo estuve en unos seminarios de transexualidad y eso jamás se tocó en la escuela (E2, Comunitaria).

2. EXPERIENCIAS EN LA FORMACIÓN: Al referirse a la formación recibida, los entrevistados mencionan experiencias valoradas en forma diversa, destacando tanto positivas como negativas.

2.1. Experiencias enriquecedoras (éticas): Recuerdo de experiencias valoradas positivamente, que constituyen un hito por la relevancia que tuvo en su formación, y que, al mismo tiempo, conllevan una dimensión ética implícita.

2.1.1. Superar prejuicios: Ese fue un gran desafio, nos tomó mucho tiempo (...) uno empieza investigando algo súper chico pero a medida que pasa el tiempo se agranda y, como estudiante de pregrado, sin experiencia en investigaciones, uno no sabe como meter tijera para cortar, entonces, en eso nos ayudaron mis profesoras guias, a cortar todo eso (...) y ahi fue que me terminé de enamorar de la diversidad y entre comillas, asumir una lucha contra los prejuicios que yo creo que en el tema de la diversidad lo que más cuesta son los prejuicios (E16, Educacional).

2.1.2. Reflexión y análisis de las propias acciones profesionales: Agregaría el ejercicio reflexivo que va más allá de una técnica, es como el constantemente estar repensando lo que se hace, y bueno creo que eso también tiene que ver un poco con la ética (Grupo 2, clinica 3).

Un momento reflexivo crítico, me pasó también que yo estuve en otra práctica primero, en un centro psicológico de una organización de diversidad sexual y duré un día, me fui porque sentía que tenían prácticas que no eran éticas (Grupo 2, Clínica 3).

También en los contextos académicos, pero que son quizás más prácticos, por ejemplo, en comunitaria cuando habia que hacer una investigación en terreno, como que ahi se da harto el tema de la ética, cuando hay que ir y encontrarse con el otro (Grupo, Clínica 2). 
2.1.3. Dimensión práctica en la formación: Mi experiencia creo que fue positiva para lo que ha sido después, creo que esa es una de las cosas positivas que tiene la escuela, darnos el espacio de la supervisión que es súper necesario (...), porque la realidad es mucho más dura que los ejemplos que te muestran y fue bueno porque el proceso que yo tuve, como psicóloga en el Centro, fue un proceso que yo pude tomar desde el principio y terminarlo (E 10, Clínica).

Mi experiencia en educacional fue una buena experiencia; yo llegué a la escuela y la escuela estaba muy desestructurada, entonces no habia un supervisor tan directo; al final llegué a trabajar a la par con las demás personas (...) entonces todo se fue construyendo en el camino y eso me permitió desempeñar funciones que a la mayoría de mis compañeros no se les abrió ese espacio para realizar (Grupo 1, Educacional).

2.1.4. Respetar al otro: Estaba fundamentalmente relacionado con el tema de la ética, respetar a los otros, dejar entre paréntesis o comillas las propias creencias, los propios valores, en relaciones que uno pueda tener a la hora de conocer a personas que se identifican o pertenecen a otras culturas (Grupo 2, Organizacional).

Es el tema de los Talleres (...) donde desde primer año, se preocuparon de sensibilizarnos en el tema de la ética profesional, el resguardo, la importancia de estar trabajando con otro, la comprensión de otro, el entendimiento, el diálogo permanente (E 11, Educacional).

2.2. Experiencias adversas (no éticas): referencias a momentos en la formación o la experiencia profesional temprana de tonalidad adversa, con frecuencia acompañadas de vivencias negativas.

2.2.1. Experiencias de discriminación, por género: Lo primero que me molestó fue 'acá no hay espacio para mujeres madres, si ustedes quieren tener hijos se tienen que salir...', y fue como: 'ya, ¿por qué?', y eso también me empezó a hacer, a cuestionarme el cómo la academia se vuelve tan ingrata a los procesos que son de la vida (E2, Comunitaria).

2.2.2. Experiencias de discriminación por color de la piel: Es una persona super-trabajadora, no cabría un espacio para crítica, en realidad las críticas no tienen que ver con la calidad de su trabajo, es sencillamente burlarse... por el color de piel (E4, Educacional).

2.2.3. Incumplimiento de la ley: El año pasado el director le pegó a un niño (...), enfrentarme a eso, qué se hace, porque lo que se decidió desde la dirección de administración fue que el suceso se iba a dar por aludido y el director pidió disculpas, pero ¿cómo enfrentas eso también?. Yo ahi tuve el tremendo conflicto moral y hasta me sentí mal porque tuve que quedarme callada y mi labor como psicóloga de la educación es velar por los niños y frente a eso yo tuve que haber hecho una denuncia (E18, Educacional)

2.2.4. Omisiones o invisibilizaciones: La situación estructural chilena de desigualdad... dentro de mi formación no estaba... si en algunos ramos de social, pero (...) es un tema que yo creo que tiene que ser transversal (E 3 , Comunitario).

2.2.5. Presiones indebidas Y personalmente me pasó que me pidieron los resultados de una persona, de una técnico paramédico, se las pidieron a mi jefe, mi jefatura me pasó la pelota a mí. Y este caballero me decía 'cómo no me puede dar los resultados' (...), le di toda la explicación que le doy a la persona y le pregunté cuál es el parentesco con ella y era la hija de la nana de un amigo de él (E8, Organizacional).

Con lo que me vi enfrentado todo el tiempo fue con el tema del diagnóstico del niños con conductas disruptivas en el aula; como trastorno antisocial... y los niños ni siquiera están en la edad de ser diagnosticados, pero en el Ministerio igual se exige un esbozo de diagnóstico (Grupo 1, Educacional).

2.2.6. Condiciones laborales no éticas: [autoridad política] les dice que las personas que están contratadas en este servicio, no debiesen estar a contrata (...) y decide arbitrariamente pasar de contrata a honorarios. Entonces, en esa situación de inestabilidad laboral, más encima se les dice que les van a cambiar el director (E17, Clínico).

Era complicado porque yo veía que se hacían cosas muy mal, como tener una mujer en crisis y que le tiren un vaso de agua y le dijeran 'vuelva cuando se sienta mejor', eso no se hace, yo vi que eso estaba muy mal; entonces era profundamente complicado éticamente decir que estaba mal, que me echaran (Grupo 1, Clínica).

3. NOCIÓN DE INTERCULTURALIDAD: Concepciones más bien circunscritas respecto de interculturalidad; centradas en algunas situaciones particulares que refieren o al contexto nacional o a experiencias en el campo de la psicología. 
3.1. Como sinónimo de poblaciones originarias: Primero la interculturalidad tiene una vinculación directa con la idea de diversidad, pero sobre todo la interculturalidad como concepto, yo la entiendo como una deuda que se mantuvo con los pueblos originarios y las expresiones indigenas, cuando alguien me dice interculturalidad yo lo entiendo así. Es como hacerse responsable de la diversidad cultural antropológica existente en el presente (E1, Comunitario).

A mí me pasa que con 'interculturales'tiendo a asociar con, siguiendo en el caso práctico, ¿qué hago yo evaluando a un niño mapuche con un test psicométrico? (E6, Clínica).

3.2. Como necesidades educativas especiales: En educacional es donde más se discuten esos temas de la diversidad, pero muy desde las necesidades educativas especiales (E2, Comunitaria).

En un ramo que aborda Necesidades Educativas Especiales (...) ahí recién se habló desde esta visión de lo más típico, que es formalmente la discapacidad, el abanico de necesidades educativas especiales (E6, Clínica).

3.3. Como diversidad, lo intercultural tiene que ver con un diálogo (...) desde los pueblos originarios hasta las expresiones culturales, digamos, urbanas, las cuales no son posturas históricas o antropológicas, sino que son culturas construidas a raíz de una serie de procesos sociales y políticos (...) la escuela también es un espacio cultural, en esta idea de la interculturalidad deberia estar el graffiti, el hip-hop (E1, Comunitario).

3.4. Como deuda: hay márgenes interculturales, incluso entre las mismas regiones, es muy distinto ser del sur que ser del norte; entonces, ahi hay un elemento que es una deuda, si me preguntan por lo intercultural, yo creo que es una deuda (...) hablamos de interculturalidad porque nunca nos hicimos cargo (E1, Comunitario).

También existen distintas culturas dentro de nosotros mismos, existe... puedes ser poblador, trabajador, estudiante, homosexual y existe una multiplicidad, pero me gustaría saber en qué minuto conversamos todos y somos lo mismo (Grupo 1, Comunitario).

4. SUGERENCIAS PARA LA FORMACIÓN EN ÉTICA E INTERCULTURALIDAD: A partir de la reflexión sobre la propia formación recibida y de experiencias profesionales tempranas surgen propuestas para una mejor preparación en ambas temáticas.

4.1. Enfatizar la reflexión: Hacer actividades que sean más autoevaluadas, que sean más reflexivas (Grupo 2, Organizacional).

4.2. Instancias prácticas: Debería haber más talleres, todos los semestres... deberíamos hacer observaciones en los colegios, observación clínica, estar más en terreno (E 18, Educacional).

Yo creo que la ética no puede estar (...) escindida de la práctica misma (E3, Comunitario).

4.3. Metodología aplicada de enseñanza-aprendizaje, incorporar, por ejemplo, videos con casos que refieran a situaciones reales que ocurren en el país e instancias de diálogo.

De repente salen en las noticias muchas cosas del conflicto mapuche, como esos casos (E18, Educacional).

En esta universidad falta más diálogos que te inviten a aunar distintas posiciones para aprender en ética más que asumir una posición defensiva o de resistencia, que así es como yo creo que aprendí ética en esta universidad, como resistencia para no dejarme pasar a llevar por las demás personas, un pensamiento beligerante (E17, Clínico).

Formación transversal: Es importante para que uno se forme como ser humano integral, y para eso no puede ser como un módulo aislado, como un electivo de esto, tiene que ser transversal (E2, Comunitaria). 
La aceptación de la diversidad en cuestión - Maria Inés Winkler, María Isabel Reyes Espejo

\section{Referencias}

1. Ballesteros A. Max Weber y la sociología de las profesiones. México (DF): Universidad Pedagógica Nacional; 2007.

2. González M, Gonzáles \& Vicencio K. Descripción del rol autopercibido del psicólogo y sus implicancias de en los procesos de formación de pregrado. Psicoperspectivas 2014; 3(1): 108-120.

3. Venegas C. Realidad laboral de la Psicología en Chile y la V Región: ¡ilibre mercado o dignidad humana?. Razón y Palabra 2010; 72. Disponible en: http://www.razonypalabra.org.mx/N/N72/Varia_72/19_Venegas_72.pdf

4. Ministerio de Salud Pública. Código Sanitario. Decreto con Fuerza de Ley No 725. Biblioteca del Congreso Nacional de Chile; 1968. Disponible en: http://www.leychile.cl/Navegar?idNorma=5595

5. Winkler MI, Pasmanik D, Alvear K, Reyes MI. Cuando el bienestar psicológico está en juego: La dimensión ética en la formación profesional de psicólogos y psicólogas en Chile. Terapia Psicológica 2007; 25(1): 5-24.

6. Winkler MI, Reyes MI. Representaciones sociales de psicólogos chilenos acerca del ejercicio profesional ético. Fundamentos en Humanidades 2006; 7(13-14): 63-89.

7. Alvear K, Pasmanik D, Winkler MI, Olivares B. ¿Códigos en la posmodernidad? Opiniones de psicólogos/as acerca del Código de Ética Profesional del Colegio de Psicólogos de Chile A. G. Terapia Psicológica 2008; 26(2): 215-228.

8. Pasmanik D, Jadue F, Winkler MI. Un acercamiento al ethos profesional en estudiantes de psicología al inicio del ciclo centrado en la formación profesional. Acta Bioethica 2012; 18(1): 111-120.

9. Winkler MI, Alvear K, Olivares B, Pasmanik D. "Lo ético es transversal y cotidiano": Dimensiones éticas en la formación y práctica en Psicología Comunitaria. Acta Bioethica 2012; 18(2): 237-245.

10. Castillo J, Winkler MI. Praxis y ética en psicología comunitaria: representaciones sociales de usuarias y usuarios de programas comunitarios en la Región Metropolitana. Psykhe 2010; 19(1): 31-46.

11. Pasmanik D, Winkler MI. Buscando orientaciones: pautas para la enseñanza de la ética profesional en psicología en un contexto con impronta postmoderna. Psykhe 2009; 18(2): 37-49.

12. Bolívar A. El lugar de la ética profesional en la formación universitaria. Revista Mexicana de Investigación Educativa 2005; 10: 93-123.

13. Cortina A. Ética aplicada y Democracia radical. Madrid: Tecnos; 2001.

14. Bilbeny N. Ética Intercultural. Barcelona, España: Ariel; 2004.

15. Wadely A, Blasco T. La ética en la investigación y la práctica psicológicas. Barcelona, España: Ariel; 1995.

16. Rodríguez N. Manual de Sociología de las profesiones. Barcelona, España: Universidad de Barcelona; 2008.

17. Ashley JM. Psychologists at Guantanamo Bay: Can their Ethical Violations be Justified? Journal of Ethics in Mental Health 2012; 7(1): 1-5.

18. Lira, E. Psicología, ética y seguridad nacional: el rol de los psicólogos. Psykhe 2008; 17(2): 5-16.

19. Ferrero A. La ética en psicología y su relación con los derechos humanos. Fundamentos en Humanidades 2000; 1(2): 17-31.

20. León F. Bioética: entre la universalidad y la interculturalidad. Los desafíos éticos de la globalización. Revista de Medicina y Humanidades 2009; 2: 1-12.

21. González M, Gonzáles E, Marín M, Martínez C. La ética intercultural: una herramienta para formar una ciudadanía cosmopolita. Frónesis 2005; 12(1): 94-109.

22. Winkler MI, Alvear K, Olivares B, Pasmanik D. Querer no basta: deberes éticos en la práctica, formación e investigación en psicología comunitaria. Psykhe 2012; 21(1): 115-129.

23. Red Psicología, Proyecto MECESUP ULS 0601. Competencias del perfil de egreso de psicólogo. En Juliá MT, (ed.). Competencias del psicólogo en Chile. Propuestas desde las universidades estatales La Serena: Editorial Universidad de La Serena; 2013: 166-188.

24. Handelsman M, Gottlieb M, Knapp S. Training ethical psychologists: An acculturation model. Professional Psychology: Research and Practice 2005; 36: 56-65.

25. Martínez-Salgado C. El muestreo en investigación cualitativa. Principios básicos y algunas controversias . Ciênc. saúde coletiva 2012; 17(3): 613-619.

26. Strauss A, Corbin J. Bases de la investigación cualitativa. Técnicas y procedimientos para desarrollar la teoría fundamentada. Bogotá: CONTUS Editorial, Universidad de Antioquia; 2002.

Recibido: 12 de junio de 2015

Aceptado: 11 de agosto de 2015 